\title{
Controlling gene expression timing through gene regulatory architecture
}

\author{
Md Zulfikar Ali ${ }^{\mathrm{a}, \mathrm{b}}$, Robert C. Brewster ${ }^{\mathrm{a}, \mathrm{b}, *}$ \\ ${ }^{a}$ Program in Systems Biology, University of Massachusetts Medical School, 368 Plantation St., Worcester, \\ MA 01605 \\ ${ }^{b}$ Department of Microbiology and Physiological Systems, University of Massachusetts Medical School, 368 \\ Plantation St., Worcester, MA 01605.
}

\begin{abstract}
Gene networks typically involve the regulatory control of multiple genes with related function. This connectivity enables correlated control of the levels and timing of gene expression. Here we study how the timing of gene expression in networks can be encoded in the regulatory DNA of a gene. Using stochastic simulations, we examine the role of binding affinity, TF regulatory function and network size in controlling the mean first-passage time to reach a fixed fraction of steady-state expression for both an auto-regulated TF gene and a target gene. We also examine how the variability in first-passage time depends on these factors. We find that both network size and binding affinity can dramatically speed up or slow down the response time of network genes, in some cases predicting more than a 100-fold change compared to constitutive expression. Furthermore, these factors can also significantly impact the fidelity of this response. Importantly, these effects do not occur at "extremes" of network size or binding affinity, but rather in an intermediate window of either quantity.
\end{abstract}

Keywords: transcriptional regulation, resource competition, network motifs, auto-regulation, gene expression

\section{Introduction}

Transcription factors (TFs) play an essential role in controlling how a cell will respond to a stimulus through the regulation of gene expression $(1 ; 2 ; 3)$. The gene regulatory code, inscribed in the DNA of each gene, specifies how the production of each gene product will be controlled in space, time and magnitude. A tremendous effort has focused on which regulatory proteins (transcription factors or TFs) regulate each gene, where they bind to accomplish their regulation and the mechanism by which the TF functions (4; $5 ; 6 ; 7 ; 8 ; 9)$. While this work has advanced our ability to read the regulatory code, an important consideration is how the timing of gene expression may also be encoded in the DNA. Although temporal patterns in expression can be arranged through a variety of mechanisms such as signalling $(10 ; 11)$ or compartmentalization $(12 ; 13 ; 14)$, here we will focus on timing encoded through transcriptional regulation. TFs are often part of a "singleinput module" network motif where one auto-regulatory TF gene controls many genes

\footnotetext{
${ }^{*}$ Corresponding author: Robert.Brewster@umassmed.edu
} 
$(15 ; 16)$; typically these target genes are consecutive enzymes along a single physiological pathway and their temporal order in expression is crucial to ensure efficient response (17; $18 ; 19 ; 20)$. However, despite the prevalence of this regulatory motif in natural genetic circuits and the importance for efficient transcriptional programs, we can not predict or design temporal gene expression patterns from this motif based on the regulatory sequence of the regulated genes.

The natural timescales to reach steady-state of gene regulatory systems is set by the decay rate of the protein product (21). In bacteria such as E. coli, where most proteins are stable, the timescale is set by the division rate of the cell. Regardless of organism, natural regulatory systems seem to operate at a wide range of timescales sometimes requiring precise tuning $(22 ; 17)$. The timing, and variability in timing, of cellular events such as metabolism and DNA damage response in $E$. coli $(17 ; 18)$, meiosis in $S$ cerevisiae $(23)$, sporulation in $B$. subtilis (24) and apoptosis in human cell lines (25) have been identified as phenomena whose temporal dynamics are driven by transcriptional regulation. The network architecture will play a role in setting these timescales and the accompanied noise (26). It is known that auto-regulation can slow down (in the case of activation) or speed up (repression) the response time of an autoregulated gene $(27 ; 21 ; 28)$. Furthermore, target genes in the network are impacted by these dynamics, each target gene in a network may have different regulatory DNA which may include differing number of TF binding sites, affinity for the TF or interactions with other TFs at those sites. Some studies have pointed to differential TF binding affinity at each target gene within a regulatory network as a driving force in setting timescales of expression $(17 ; 18 ; 29 ; 30 ; 31 ; 32 ; 33 ; 34)$. However, other factors such as the size of the network (number and affinity of additional binding sites) likely play a significant role; TFs can target dozens to hundreds of specific sites in the genome and the presence of competitive sites can have a significant impact on the steady-state level of expression and noise of the genes in the network $(35 ; 36 ; 37 ; 38 ; 39 ; 40 ; 41)$. Exploring the role regulatory architecture and competing binding sites play in tuning not only the average response time but also the precision of that response is an open question.

Here, we use stochastic simulations to characterize how a multitude of factors control the timing of gene expression (42). The primary focus of this work is auto-regulated TF genes and the target genes they regulate. The majority of TFs in E. coli are auto-regulated, and as such it is crucial to study this motif and its role in regulating the dynamics of gene expression of both itself and the other genes under its control within the network $(4 ; 5)$. We start by examining auto-regulated genes alone and explore how details of the regulation such as binding affinity, TF regulatory role (activation or repression) and network size (number of TF binding sites) influence the mean first-passage time (MFPT) to reach $50 \%$ of the steady state expression level. Importantly, the MFPT is a single-cell measurement of response time; as opposed to measuring how long the bulk takes to reach a certain expression level on average. MFPT has been widely used to study biological processes $(27 ; 43 ; 44 ; 45 ; 46)$. Furthermore, we also examine the robustness of the response; i.e. the variability in firstpassage time at the single cell level; auto-regulation is known to impact the stability and noise of expression $(47 ; 48)$, here we explore how these elements influence the variability of response of a gene within the network. 


\section{Results}

\section{Model}

The general model of gene expression for a self regulatory gene (TF-gene) is outlined in Fig. 1A. In this model, the TF-gene produces TFs that can bind to the promoter of the TF-gene with probability set by the binding-rate $\left(k_{\mathrm{on}}\right)$. The TF binding affinity is controlled through the unbinding rate $\left(k_{\text {off }}\right)$, which is related to the sequence specificity of the binding site; higher affinity means lower unbinding rate and vice versa (49). The regulatory function of the $\mathrm{TF}$ is represented as a change to transcription rate when the $\mathrm{TF}$ is bound; the TF-gene transcribes at rate $r$ when bound by a TF and $r_{0}$, when unoccupied. The ratio of the transcribing rates $\left(\alpha=r / r_{0}\right)$ for the TF-gene determines whether the gene is self-activating $(\alpha>1)$, self-repressing $(\alpha<1)$ or constitutive $(\alpha=1)$. In later sections, we add the possibility of decoy TF binding sites to the model. Outlined in Fig. 1B, decoy TF binding sites bind a TF (thus making it unavailable to bind to the TF gene) but do not result in regulation (or do not regulate a gene whose product we consider). Decoy sites have the same TF binding rate as the TF gene but can have a distinct unbinding rate, $k_{\text {off,d }}$. For the sake of simplicity we consider $N$ identical decoy sites, though in a real network these sites could have a spectrum of affinities. Finally, as outlined in Fig. 1C, we will explore the dynamics of a target gene. As is the case for decoys, the TF binding site for this gene binds TFs with the same rate as every other binding site and has a distinct unbinding rate, $k_{\mathrm{off}, \mathrm{t}}$. We also consider that the regulatory role of the TF may be different on this target gene and thus we define $\alpha_{t}$ as the ratio of TF-bound $\left(r_{t}\right)$ over TF-unbound transcription rate $\left(r_{0, t}\right)$ of this gene.

To explore the dynamics of this system, we employ a stochastic simulation algorithm to compute mean first passage time (MFPT) for gene expression to reach half of the steady state expression level $(43 ; 50)$. The choice of threshold, $50 \%$ of the steady state expression, is arbitrary, but consistent with other work in the field $(28 ; 51 ; 52)$, and the consequence of this choice is also discussed at points below. As demonstrated schematically in Fig. 1D, at time $t=0$ the network is switched on and the genes start to express until the threshold is achieved. The time to reach the threshold is termed as "first passage time" (FPT) and is computed for each of $>10^{5}$ individual trajectories (cells). The MFPT is obtained by taking the mean of the FPT distribution. We also calculate the noise in the timing of response represented by the $\mathrm{CV}$ which is the ratio of the standard deviation to the mean of the FPT distribution.

\section{Role of TF-binding affinities in controlling expression timing of auto- regulated genes}

We start by investigating the impact of TF-binding affinity in auto-regulatory motifs without any competing binding sites (decoy, Fig. 1B) or target genes (Fig. 1C). For both positive and negative auto-regulation motifs, the TF-binding affinity $\left(k_{\text {off }}\right)$ impacts the mean first passage time (MFPT) to reach half of the Steady-state expression level (SSE) in a nonmonotonic way. In Fig. 2A, we show the dependence of the MFPT on the binding affinity of a positive auto-regulatory gene. In the two extreme limits of affinity, the MFPT is a minimum approximately equal to that for a constitutive gene (set by the transcription and translation rates, roughly equal to one cell-cycle here; see supplementary Fig. S1A). When 
the binding affinity is very weak, the promoter is always free and expresses with a lower basal transcription rate $r_{0}$ and thus the MFPT is essentially the same as a constitutive gene with transcription rate $r_{0}$. On the other hand, when the binding affinity is strong, the promoter is always occupied by a TF and as such the MFPT is similar to a constitutive gene with transcription rate $r$. However, the MFPT is slightly higher than that of a constitutive gene due to the fact that the first binding event (to initiate the auto-activation) takes additional time and thus the MFPT is the sum of the MFPT of a constitutive gene and the average time to express the first $\mathrm{TF}$ to initiate the auto-activation. Interestingly, between these two extremes, the MFPT has an intermediate maximum at a specific value of $\mathrm{TF}$ binding affinity $\left(k_{\text {off }}\right)$. The height of this intermediate maximum depends on the strength of the activation $\left(\alpha=r / r_{0}\right)$, with lower strength corresponding to smaller maxima. This maxima indicates that in a specific range of binding affinity, the auto-activating TF gene can respond substantially slower than a constitutive gene, taking several generations to respond and reach the half maxima of steady-state expression. For a given $\alpha$, altering translation rate shifts the position of maxima without affecting the peak MFPT (Fig. 2A, dashed curves). This shift vanishes if we replot the data as a function of steady state TFoccupancy rather than $k_{\text {off }}$ (Fig. $2 \mathrm{~B}$ ); when plotted as a function of TF-occupancy the data for different translation rates collapses such that the maxima occurs at a specific value of TF occupancy for a given $\alpha$ regardless of the translational dynamics. However, the occupancy level where the maxima occurs still has a dependence on the strength of regulation, $\alpha$.

The non-monotonic behavior of the MFPT for the auto-activating gene can be understood by examining the relationship of two different quantities with $k_{\text {off }}$ : the TF-production rate from a single TF $(R)$ and the Steady state expression level (SSE). In this case, SSE is proportional to how much TF must be made by the gene to reach the FPT threshold and $R$ is an approximation for the speed in which the system approaches that level (SSE has units of number of proteins and $R$ is a rate of production); in practice $R$ depends on time in this system and changes as the number of TFs increases, but we use this simplified rate of expression to gain intuition about the system. As such, the ratio of these quantities ( $\mathrm{SSE} / R)$ should tell us approximately the timescale of response of the gene. For the auto-activating gene, Fig. $2 \mathrm{C}$ shows the steady state expression level (blue curve) and the quantity $1 / R$ (red curve) as a function of TF binding affinity, $k_{\text {off }}$. The steady state expression level decreases as the binding affinity is increased, from a maximal value of SSE $=r b / \gamma_{m} \gamma$ for a very strong affinity when the promoter is always occupied by a TF to SSE $=r_{0} b / \gamma_{m} \gamma$ for very weak activation. The production rate $R$ behaves in a similar fashion and we plot $1 / R$ as a red curve on the same axis. The response time, which is then the product of the red and blue curves $(\mathrm{SSE} / R$ ) will be non-monotonic with an intermediate maximum that approximately coincides with the maximum of the MFPT; although the maximum does not exactly coincide due to the simplifications in this calculation.

As shown in Fig. 2D, we see a similar phenomenon for an auto-repressing gene. In all cases, the MFPT is a minimum value, less than a cell-cycle (28), when the TF off-rate is very low (high affinity) and approaches the MFPT of a constitutive gene when the site is very weak. For auto-repression we once again see non-monotonic behavior with a minimum in MFPT less than one cell-cycle at an intermediate value of $k_{\text {off }}$ (Fig. 2D blue and red curves). As the auto-regulatory strength, $\alpha$ is made smaller (stronger repression) the value of $k_{\text {off }}$ at the minimum also gets smaller. When repression is "complete" $(\alpha=0)$, i.e. 
the transcription rate is 0 when bound by repressor, the intermediate minimum disappears (Fig. 2D, yellow curve). In Fig. 2E, we once again see that the MFPT is collapsed by plotting MFPT as a function of TF occupancy. We also see, like in auto-activation, the quantity SSE/ $R$ captures the non-monotonic behavior of the MFPT of an auto-repressing gene as well (Fig. 2F).

For an auto-activating gene, we can also examine how the magnitude of the peak in MFPT and the corresponding off-rate at the peak depends on the strength of autoactivation. We find that the peak in MFPT has a power-law dependence on auto-regulatory strength $\alpha$ and the power-law exponent is independent of the transcription and translation rates (see Fig. 3A). In this case, the exponent to the power-law is approximately 0.5 , meaning for example that a 4 -fold increase in the strength of activation will net a 2 -fold increase in the peak MFPT. However, the arbitrary choice for MFPT threshold affects the powerlaw exponent in a monotonic way with lower thresholds having a slightly higher exponent and vice versa (Fig. 3B,C). Furthermore, the off-rate $\left(k_{\text {off }}\right)$ corresponding to the MFPT peak collapses to a single power-law curve when scaled with basal expression rate, $r_{0} b$ (see Fig. 3D). In this case, the power-law exponent is approximately 1, and as in our example above a 4 -fold increase in activation strength would have a peak in the MFPT when the normalized off-rate is increased approximately 4 -fold. Once again, the power-law exponent depends on the chosen value of the MFPT threshold and the exponent is higher for lower threshold and decreases monotonically as the threshold is increased (Fig. 3E,F). Furthermore, the MFPT for a constitutive gene can be estimated from the power-law fit by setting $\alpha=1$. We plot the estimated MFPT and the MFPT computed from simulation for varying parameter ranges in Fig. S1B and find comparable results. For auto-repressing genes, the minima in MFPT as well as the off-rates corresponding to the minima deviate from this power-law behavior (see Supplementary Fig. S3 A,B).

\section{The role of TF competition in controlling expression timing}

Next, we investigate the effect of TF sharing with competing binding sites (decoy). Competing binding sites for the $\mathrm{TF}$ reduce the availability of free $\mathrm{TF}$ ( $\mathrm{TF}$ not bound to any gene or competing binding sites). As a consequence, both the steady state expression (SSE) and MFPT is affected. As shown in Fig. 4A, the presence of competing binding sites in an auto-activating TF gene has effects similar to varying the TF-binding affinity in that the MFPT once again shows non-monotonic behavior. However, in this case the peak value of the MFPT can be significantly higher. The magnitude of the maxima depends on TF binding affinity with weaker sites displaying higher peaks (Fig. 4A red and blue curves). The red curve in Fig. 4A has an affinity chosen to be consistent with the strong LacI binding site lacIO1 (38) and we see a predicted maximum in MFPT of 29 cell cycles. The maximum in MFPT becomes higher for weaker binding sites and can reach hundreds of cell-cycles for sites with affinities consistent with lacO2 $\left(k_{\text {off }} \sim 0.01 s^{-1}\right)$ or lacO3 $\left(k_{\text {off }} \sim 0.6 s^{-1}\right)$. This can be seen in Fig. $4 \mathrm{C}$, where we plot a heatmap of MFPT in number of competing binding sites and affinity space. These timescales are exceptionally long compared to, for instance, the total duration of log phase growth of $E$. coli in lab conditions which is typically less than 10 generations. This implies that for specific "network sizes", the TF gene could not reach a significant fraction of its SSE level in a realistic time frame. The peaked MPFT behavior start to disappear as the affinity is further reduced (Fig. 4C). 
To understand the non-monotonic behavior of the MFPT as a function of decoy site number, we plot the steady state expression (SSE) level, the number of free TFs and the number of TF-bound decoy sites in Fig. 4B inset. Once again we will argue based on the balance between the SSE level (a fraction of which must be achieved to achieve the FPT) and the rate at which it gets there, which is a function of the number of free TFs (i.e. TFs not already bound to binding sites). When the decoy number is small (less than the constitutive expression level of roughly 390 proteins for this example), the presence of decoy sites do not alter the SSE significantly (solid blue line). However, in this regime, the free TF number reduces linearly with decoy number as the TFs bind to the available decoy sites (dashed line in Fig. 4B inset). This reduction in free $\mathrm{TF}$ will slow the production rate of $\mathrm{TF}$ and the MFPT will increase with decoy number in this regime. This decrease in free TF stops when the number of decoys is greater than the constitutive expression level, at this stage, very few if any TFs are free and the production rate will be roughly insensitive to decoy number. The SSE level in this regime will now decrease rapidly with decoy number signifying an overall decrease in the MFPT. The intermediate maxima will then occur approximately when the number of decoys and SSE levels of TF are comparable. It can also be seen from the heatmap (Fig. 4C) and Fig. 4A that that the number of decoys corresponding to the peak MFPT reduces as the affinity is lowered; low affinity also corresponds to lower SSE level for TFs. Unlike when affinity is varied, we do not find power-law behavior for the peak MFPT as a function of regulatory strength when decoys are varied (see Supplementary Fig. $\mathrm{S} 3 \mathrm{C}, \mathrm{D})$.

For a self-repressing gene, we find that with increasing decoy sites, the MFPT first decreases, attains a minimum value and then again goes up asymptotically reaching the MFPT for a constitutive gene with basal rate $r_{0}$ (Fig. 4D). In Fig. 4D, we see that at zero occupancy (achieved at high decoy numbers) the MFPT is approximately equal to the MFPT of a constitutive gene (black dashed line). Decreasing the decoy number increases the occupancy down to a minimum which is always less than one and is set by the TFbinding affinity and production rates. The MFPT at this point depends on TF-binding affinity which was shown in Fig. 2D and Fig. 4D. For complete auto-repression (Fig. 4D, dashed blue curve) the MFPT at zero decoy is close to 0.21 cell cycle consistent with (28). Interestingly, even for complete repression we see a slight dip in MFPT as decoys are introduced. In Fig. 4F, we show a heat map of MFPT in number of competing binding sites and affinity space. For $\alpha=0.1$ the heat map shows a global minimum in MFPT corresponding to $k_{\text {off }}=0.009 \mathrm{~s}^{-1}$ and 80 decoys. Moreover, the qualitative behaviors are consistent for different parameter sets (see Fig. S2, where we have used cell division time of $38.5,58$ and $116 \mathrm{~min})$.

\section{The precision of expression timing depends on the nature of regulation}

Next, we investigate the effect of TF-binding affinity and decoy size in controlling the noise of first passage time. We use coefficient of variation (CV) defined as the ratio of the standard deviation of first passage time and MFPT as a measure of noise. In Fig. 5C,F, we plot CV of first passage time versus MFPT for auto-activation or auto-repression if either the TF binding affinity or the number of decoys is used as a control parameter of the system. We know from Fig. 2 that for auto-activation the MFPT will first rise and then fall as the affinity is increased, Fig. 5C shows that despite degeneracy in MFPT (two different $k_{\text {off }}$ 
values give the same MFPT), the associated noise in the MFPT is different for strong and weak binding sites with stronger binding leading to tighter distributions around the mean (Fig. 5A). For auto-repression (Fig. 5B,C) the story is similar but reversed; the MFPT is now less than 1 and the noise in MFPT is lower for weak binding compared to strong binding site having same MFPT. When number of decoys is used as a control parameter, the CV versus MFPT has trend qualitatively similar to affinity as the control parameter (Fig. 5F). In this case, zero competition (no decoy) leads to the minimum CV for auto-activation and maximum CV for auto-repression (Fig. 5D,E). Interestingly, for auto-repressing gene having very strong binding affinity, the FPT distribution is bimodal (Fig. 5B, bottom curve) and disappears for lower affinities. In all other scenarios, the distribution is unimodal.

\section{The expression timing of a target gene depends on the nature of TF reg- ulation}

In this section, we explore the impact of a TF's mode of auto-regulation on the expression timing of a target gene. The TF gene can be expressed in three different ways: autoactivated $(\alpha>1)$, auto-repressed $(\alpha<1)$, and constitutively expressed $(\alpha=1)$. In Fig. 6 $\mathrm{A}$ and B, we plot the MFPT of an activated (Fig. 6A) or repressed (Fig. 6B) target gene as a function of TF off-rate at the target promoter. The colored lines in the plot represent different modes of auto-regulation of the TF (different values of $\alpha$ ). In this plot, the number of total TFs is kept constant by adjusting the translation rate to counteract the effect of changing $k_{\text {off. }}$.

As the TF-target affinity is decreased the MFPT increases irrespective of the TF autoregulatory mode or the type of regulation of the target gene (activation, Fig. 6A or repression, Fig. 6B). For target repression, a lower TF-target affinity corresponds to higher target expression and thus a higher MFPT. On the other hand for target-activation a lower TF-target affinity reduces the target expression but at the same time the rate of production of target is also hampered due to lowering of the affinity which is more substantial. As a result the MFPT of the target gene increases with lowering affinity in case of activation. One interesting feature revealed from Fig. 6A,B is that the MFPT curves for different autoregulatory strength of the TF gene $(\alpha)$ never intersect each other. The MFPT curve of a target gene regulated by an auto-repressed TF gene is always greater than the MFPT of a target gene under the control of an auto-activated TF gene and the constitutive TF gene defines the boundary of separation between the two cases (Fig. 6B). We get the opposite behavior when the target gene is activated by a TF gene. In this case, the MFPT curve of target gene regulated by an auto-repressed TF gene is always lower than the MFPT of target gene under the control of an auto-activated TF gene with the constitutive TF gene defining the separation between the two (Fig. 6A). This tells us that timing of expression of the TF-gene is the key factor for determining the timing of expression of target gene under control of the TF gene. To further explore this relationship, we plot the MFPT of a target gene as a function of MFPT of the TF-gene which regulates the target gene (Fig. 6C). We find that for target activation, the MFPT of the TF and target gene are positively correlated whereas for target repression it is anti-correlated. A useful way to view this data is to scale the MFPTs by the respective MFPT for constitutive TF expression $(\alpha=1)$. This is shown in Fig. 6C (also see Supp. Fig. S4A), where we see the MFPT-MFPT phase space is separated into four quadrants; 1) self-repressed gene repressing a target (RR), 2) 
self-repressed gene activating a target (RA), 3) self-activated gene repressing a target (AR), and 4) self-activated gene activating a target (AA). It further provides us with a tool to identify the mode of TF-gene by simply comparing the MFPT of a target gene regulated by an auto-regulated TF gene and MFPT of the same target gene regulated by a constitutive TF gene. For example, in case of target activation, MFPT of target gene under the control of auto-regulatory TF gene higher than that of under constitutive TF gene means that the $\mathrm{TF}$ gene is auto-activated and vice versa.

We further explore the expression timing relationship when the binding sequence for $\mathrm{TF}$ and target genes are exactly identical, which means the binding affinity of TFs being identical for both TF and target gene. In our previous work (38), we have shown that an auto-repressed gene and its target gene have different steady state expression levels even though their binding sequences are exactly identical. In Fig. 6D, we plot the MFPTs of the target gene which is regulated by an auto-regulated TF gene versus MFPT of the TF gene, the regulation being identical for both $\mathrm{TF}$ and target gene. Each curve is generated by tuning the $\mathrm{TF}$ affinity for $\mathrm{TF}$ and target gene while keeping other parameters constant. We find that the target gene is always slower than the $\mathrm{TF}$ gene to reach half of their respective steady state expression for negative regulation, i.e., target gene repressed by an auto-repressed TF gene (Fig. 6D, open squares. Also, see Supp Fig. S4B, where number of decoy sites are varied keeping the affinity fixed). The difference in their timing is maximum for high affinity binding sites and approaches to one-to-one line showing similar timing in expression as the affinity is lowered. For target gene activated by an auto-activated TF gene, we find that the target gene is faster than the TF gene, however, the difference is not significant (Fig. 6D, filled circles).

\section{Discussion}

The decay rate of proteins sets the natural timescale of the response of transcriptional networks (21); in bacteria where proteins are long-lived this timescale tends to be the division rate of the organism. Although specific network connections are known to speed or slow this response by several fold, extremely slow or fast responses have been observed in a growing number of systems $(22 ; 25 ; 23 ; 24)$. Here, we systematically investigate the regulatory features that control gene expression timing of auto-regulated TFs, specifically in determining the mean first-passage time (MFPT) of reaching half of the steady-state level of expression. Interestingly, we find that auto-activation and auto-repression, around a specific range of TF binding affinities, are capable of slowing or speeding response times by orders of magnitude. Similarly, we find that extra TF binding sites, can alter the timing response in a more dramatic fashion. These extra binding sites may serve to regulate other promoters in the network or they may serve no specific function (i.e. decoys). Crucially, this means that for networks of particular size, a gene may not be realistically able to reach steady-state levels in physiologically reasonable timescales, in some extreme cases requiring hundreds of generations simply to reach half of the steady-state level. Despite arguments of the inherent interest of "steady-state levels" in such networks the concept is essentially irrelevant.

We also examine how the fidelity of single-cell timing depends on these physical parameters. Due to the intermediate extrema in MFPT, there are typically two values of 
TF binding affinity or decoy number that produce an identical "mean response", however these two regulatory parameter sets produce significantly different variance in timing among individual cells. As a result, for the majority of achievable MFPTs of an auto-regulatory gene there is both a "quiet" and "noisy" way to achieve a given response time. For autoactivation, higher off-rates and larger numbers of decoys produce noisier responses while the opposite is true for auto-repression. Just like for the case of average gene expression levels where it is argued that the ability to tune noise independent of mean can be important for fitness, it stands to reason the same holds for the speed of response of a gene's transcriptional response and thus the ability to reach similar average response times with very different levels of noise can be useful. Finally, we showed how the response of a target gene is controlled by the mode and details of regulation of the TF gene. Interestingly, for any mode of target regulation (activation or repression) the target gene MFPTs are well distinguished for different modes of TF gene regulation.

Overall, understanding the molecular features that dictate the timing in expression for genes is an important part of reading natural gene networks and designing efficient expression timing for synthetic networks.

\section{Acknowledgments}

We wish to thank Vinuselvi Parisutham, Sunil Guharajan and Sandeep Choubey for helpful discussions. Research reported in this publication was supported by NIGMS of the National Institutes of Health under award R35GM128797.

\section{Materials and Methods}

\section{Model and Simulation methodology}

Here, we describe a generalized model for an auto-regulatory TF-gene, one target gene and multiple competing binding sites. The TF-gene product, $\mathrm{TF}$ protein $(\mathrm{X})$, binds to its own promoter $\left(P_{\mathrm{TF}}\right)$, to the promoter of the target gene $\left(P_{\text {Target }}\right)$, and to the decoy sites $(N)$ with a constant rate $k_{\text {on }}$ per free TF per unit time. TF unbinds from the promoter with a sequence dependent off-rate $\left(k_{\text {off }}\right)$ which determines the TF-binding affinity of the binding site. The off-rates of a bound TF from the promoters of the TF and target, and from the decoy sites are $k_{\mathrm{off}}, k_{\mathrm{off}, \mathrm{t}}$, and $k_{\mathrm{off}, \mathrm{d}}$ per unit time, respectively. mRNAs are produced at the rate $r_{0}$ from a $\mathrm{TF}$-free promoter (basal transcription) and rate $r$ from a TF-bound promoter. The ratio of $r_{0}$ and $r$ determines whether the gene is repressed $\left(r / r_{0}<1\right)$ or activated $\left(r / r_{0}>1\right)$. This ratio is termed auto-regulatory strength, $\alpha$. Furthermore, $\alpha=1$ describes a constitutive gene. mRNAs are translated into protein at the rate $b$. The mRNAs and the proteins are degraded at the rate $\gamma_{\mathrm{m}}$ and $\gamma$, respectively. The lifetime of protein in E. coli and many other organisms are much longer, typically 2-3 hrs, and hence the protein degradation, $\gamma=\ln (2) / \tau$, is assumed to be due to dilution during cell division $(\tau)$. We further assume that all proteins (free protein, TF bound to promoter and TF bound to decoy sites) degrades with the same rate. The set of reactions describing the model above are listed below. 


$$
\begin{aligned}
& m_{\mathrm{TF} \text { (Target) }} \stackrel{r_{0}}{\rightarrow} m_{\mathrm{TF} \text { (Target) }}+1 \quad \text { (Production of mRNA from TF free promoter) } \\
& m_{\mathrm{TF} \text { (Target) }} \stackrel{r}{\rightarrow} m_{\mathrm{TF} \text { (Target) }}+1 \quad \text { (Production of mRNA from TF bound promoter) } \\
& m_{\mathrm{TF}} \stackrel{b}{\rightarrow} m_{\mathrm{TF}}+X \quad \text { (Translation of TF protein) } \\
& m_{\text {Target }} \stackrel{b}{\rightarrow} m_{\text {Target }}+Y \quad \text { (Translation of target protein) } \\
& X+P_{\mathrm{TF}(\text { Target })} \stackrel{k_{\mathrm{on}}}{\longrightarrow} X P_{\mathrm{TF} \text { (Target) }} \quad \text { (Binding of a TF to a promoter) } \\
& X P_{\mathrm{TF} \text { (Target) }} \stackrel{k_{\mathrm{off}, \mathrm{TF}(\text { Target) }}}{\longrightarrow} X+P_{\mathrm{TF} \text { (Target) }} \quad \text { (Unbinding of a TF from a promoter) } \\
& m_{\mathrm{TF}} \stackrel{\gamma_{\mathrm{m}}}{\longrightarrow} m_{\mathrm{TF}}-1 \quad \text { (Degradation of mRNA) } \\
& m_{\mathrm{TF} \text { (Target) }} \stackrel{\gamma_{\mathrm{m}}}{\longrightarrow} m_{\mathrm{TF}(\text { Target })}-1 \quad \text { (Degradation of mRNA) } \\
& X+N \stackrel{k_{\mathrm{on}}}{\longrightarrow} X N \quad \text { (Binding of a TF to decoy) } \\
& X N \stackrel{k_{\text {off,Decoy }}}{\longrightarrow} X+N \quad \text { (Unbinding of a TF from decoy) } \\
& X, Y \stackrel{\gamma}{\rightarrow} \phi \quad \text { (Degradation of TF protein) } \\
& X P_{\mathrm{TF} \text { (Target) }} \stackrel{\gamma}{\rightarrow} P_{\mathrm{TF} \text { (Target) }} \quad \text { (Degradation of } \mathrm{TF} \text { from promoter-complex) } \\
& X N \stackrel{\gamma}{\rightarrow} N \quad \text { (Degradation of TF bound to Decoy) }
\end{aligned}
$$

We implement the simulations for stochastic reaction systems using Gillespie's algorithm (42) in C programming, to compute mean first passage time (MFPT). The threshold for MPFT is set to $50 \%$ of steady state expression (SSE) unless specified. Simulations are run for sufficiently long time $\left(\sim 10^{6} \mathrm{~s}\right)$ to get rid of the transient dynamics and then steady state distributions (TF and target protein expressions) are obtained by sampling over time with a time interval $\left(T_{\mathrm{S}}\right)$, long enough for the slowest reaction to occur 20 times on average $\left(T_{\mathrm{S}}\right.$ $=20$ over rate for slowest reaction). Next, we run the simulations using the steady state expressions as input to obtain gene expression trajectories. At time $t=0$, the network is switched on to express until the expression reaches a threshold. The time for each trajectory to reach that threshold is termed as first passage time and their mean is referred as mean first passage time (MFPT). MFPT and noise in first passage times are computed using at least $10^{5}$ individual trajectories. For simulations, we use $k_{\text {on }}=0.0027 / s((53))$, $\gamma_{\mathrm{m}}=0.011 / \mathrm{s}((54))$ and $\gamma=0.0001,0.0002,0.0003 \mathrm{~s}^{-1}$. These rates correspond to $90 \mathrm{sec}$ average life for mRNA and a cell-cycle of 115,56 and $38.5 \mathrm{~min}$, respectively. $k_{\text {off }}$ is varied in a range of $0.0002-100$ per TF per second to cover the binding affinities corresponding to a wide varieties of natural and synthetic promoters in E.coli such as lacOID, lacO1, lacO2, lacO3. Typical transcription rate in E. coli is of the order of $0.33 \mathrm{~s}^{-1}$ for constitutive gene (55), as such we use a basal rate in a range $r_{0}=0.001-0.05 \mathrm{~s}^{-1}$ such that the ten -fold activation correspond to a transcription rate of $r_{0}=0.01-0.5 \mathrm{~s}^{-1}$. Protein numbers in $E$. coli varies from few units to thousands (56) as such we vary translation rate $(b)$ in a range of $0.025-0.1 s^{-1} \mathrm{mRNA}^{-1}$. 


\section{References}

[1] jacob F, Monod J. Genetic regulatory mechanisms in the synthesis of proteins. J Mol Biol. 1961;3:318-356.

[2] Ptashne M. A genetic switch: phage lambda revisited. 3rd ed. Cold Spring Harbor, N.Y.: Cold Spring Harbor Laboratory Press; 2004.

[3] Mitchell PJ, Tjian R. Transcriptional regulation in mammalian cells by sequencespecific DNA binding proteins. Science. 1989;245(4916):371-378.

[4] Santos-Zavaleta A, Salgado H, Gama-Castro S, Sánchez-Pérez M, Gómez-Romero L, Ledezma-Tejeida D, et al. RegulonDB v 10.5: tackling challenges to unify classic and high throughput knowledge of gene regulation in E. coli K-12. Nucleic Acids Res. 2019;47(D1):D212-D220.

[5] Karp PD, Ong WK, Paley S, Billington R, Caspi R, Fulcher C, et al. The EcoCyc Database. EcoSal Plus. 2018;8(1).

[6] Hermsen R, Tans S, ten Wolde PR. Transcriptional regulation by competing transcription factor modules. PLoS Comput Biol. 2006;2(12):e164.

[7] Carey LB, van Dijk D, Sloot PMA, Kaandorp JA, Segal E. Promoter Sequence Determines the Relationship between Expression Level and Noise. PLOS Biology. 2013;11(4):1-15. doi:10.1371/journal.pbio.1001528.

[8] Kosuri S, Goodman DB, Cambray G, Mutalik VK, Gao Y, Arkin AP, et al. Composability of regulatory sequences controlling transcription and translation in Escherichia coli. Proc Natl Acad Sci USA. 2013;110(34):14024-14029.

[9] Sharon E, Kalma Y, Sharp A, Raveh-Sadka T, Levo M, Zeevi D, et al. Inferring gene regulatory logic from high-throughput measurements of thousands of systematically designed promoters. Nat Biotechnol. 2012;30(6):521-530.

[10] Pedraza JM, Paulsson J. Effects of molecular memory and bursting on fluctuations in gene expression. Science. 2008;319(5861):339-43.

[11] Di-Bella JP, Colman-Lerner A, Ventura AC. Properties of cell signaling pathways and gene expression systems operating far from steady-state. Scientific Reports. 2018;8. doi:10.1038/s41598-018-34766-0.

[12] Boija A, Klein IA, Sabari BR, Dall'Agnese A, Coffey EL, Zamudio AV, et al. Transcription Factors Activate Genes through the Phase-Separation Capacity of Their Activation Domains. Cell. 2018;175:1842-1855. doi:10.1016/j.cell.2018.10.042.

[13] Lee REC, Walker SR, Savery K, Frank DA, Gaudet S. Fold change of nuclear NFB determines TNF-induced transcription in single cells. Mol Cell. 2014;53:867-879. doi:10.1016/j.molcel.2014.01.026. 
[14] Lu Y, Wu T, Gutman O, Lu H, Zhou Q, Henis YI, et al. Phase separation of TAZ compartmentalizes the transcription machinery to promote gene expression. Nature Cell Biology. 2020;22:453-464. doi:10.1038/s41556-020-0485-0.

[15] Shen-Orr SS, Milo R, Mangan S, Alon U. Network motifs in the transcriptional regulation network of Escherichia coli. Nat Genet. 2002;31(1):64-8.

[16] Lee TI, Rinaldi NJ, Robert F, Odom DT, Bar-Joseph Z, Gerber GK, et al. Transcriptional regulatory networks in Saccharomyces cerevisiae. Science. 2002;298(5594):799804 .

[17] Zaslaver A, Mayo AE, Rosenberg R, Bashkin P, Sberro H, Tsalyuk M, et al. Just-intime transcription program in metabolic pathways. Nat Genet. 2004;36(5):486-491.

[18] Friedman N, Vardi S, Ronen M, Alon U, Stavans J. Precise temporal modulation in the response of the SOS DNA repair network in individual bacteria. PLoS Biol. $2005 ; 3(7): \mathrm{e} 238$.

[19] Ronen M, Rosenberg R, Shraiman BI, Alon U. Assigning numbers to the arrows: parameterizing a gene regulation network by using accurate expression kinetics. Proc Natl Acad Sci U S A. 2002;99(16):10555-60.

[20] Arnone MI. Bringing Order to Organogenesis. Nature Genetics. 2002;30(4):348-350. doi:10.1038/ng0402-348.

[21] Alon U. An introduction to systems biology: design principles of biological circuits. Chapman \& Hall/CRC mathematical and computational biology series. Boca Raton, FL: Chapman \& Hall/CRC; 2007.

[22] Yurkovsky E, Nachman I. Event timing at the single-cell level. Brief Funct Genomics. 2013;12:90-98. doi:10.1093/bfgp/els057.

[23] Nachman I, Regev A, Ramanathan S. Dissecting timing variability in yeast meiosis. Cell. 2007;131:544-556. doi:10.1016/j.cell.2007.09.044.

[24] Fujita M, Losick R. Evidence that entry into sporulation in Bacillus subtilis is governed by a gradual increase in the level and activity of the master regulator Spo0A. Genes dev. 2005;19:2236-2244. doi:10.1101/gad.1335705.

[25] Spencer SL, Gaudet S, Albeck JG, Burke JM, Sorger PK. Non-genetic origins of cell-to-cell variability in TRAIL-induced apoptosis. Nature. 2009;459:428-432. doi:10.1038/nature08012.

[26] Eldar A, Elowitz MB. Functional roles for noise in genetic circuits. Nature. 2010;467(7312):167-73.

[27] Co AD, Lagomarsino MC, Caselle M, Osella M. Stochastic timing in gene expression for simple regulatory strategies. Nucleic Acids Res. 2017;45(3):1069-1078.

[28] Rosenfeld N, Elowitz MB, Alon U. Negative autoregulation speeds the response times of transcription networks. J Mol Biol. 2002;323(5):785-793. 
[29] Culyba MJ, Kubiak JM, Mo CY, Goulian M, Kohli RM. Non-equilibrium repressor binding kinetics link DNA damage dose to transcriptional timing within the SOS gene network. PLoS Genet. 2018;14(6):e1007405.

[30] Kalir S, McClure J, Pabbaraju K, Southward C, Ronen M, Leibler S, et al. Ordering genes in a flagella pathway by analysis of expression kinetics from living bacteria. Science. 2001;292(5524):2080-3.

[31] Gaudet J, Mango SE. Regulation of organogenesis by the Caenorhabditis elegans FoxA protein PHA-4. Science. 2002;295(5556):821-825.

[32] Yosef N, Regev A. Impulse control: temporal dynamics in gene transcription. Cell. 2011;144(6):886-896.

[33] Gao R, Stock AM. Temporal hierarchy of gene expression mediated by transcription factor binding affinity and activation dynamics. mBio. 2015;6(3):e00686-00615.

[34] Kuchina A, Espinar L, Çağatay T, Balbin A, Zhang F, Alvarado A, et al. Temporal competition between differentiation programs determines cell fate choice. Mol Syst Biol. 2011;7:557.

[35] Burger A, Walczak AM, Wolynes PG. Abduction and asylum in the lives of transcription factors. Proc Natl Acad Sci USA. 2010;107(9):4016-4021.

[36] Brewster RC, Weinert FM, Garcia HG, Song D, Rydenfelt M, Phillips R. The transcription factor titration effect dictates level of gene expression. Cell. 2014;156(6):1312-1323.

[37] Bokes P, Singh A. Protein copy number distributions for a self-regulating gene in the presence of decoy binding sites. PloS one. 2015;10(3):e0120555. doi:10.1371/journal.pone.0120555.

[38] Ali MZ, Parisutham V, Choubey S, Brewster RC. Inherent regulatory asymmetry emanating from network architecture in a prevalent autoregulatory motif. Elife. 2020;9.

[39] Das S, Choubey S. Tunability enhancement of gene regulatory motifs through competitionfor regulatory protein resources. Phys Rev E. 2020;102(5):052410. doi:10.1103/PhysRevE.102.052410.

[40] Lee TH, Maheshri N. A regulatory role for repeated decoy transcription factor binding sites in target gene expression. Mol Syst Biol. 2012;8:576.

[41] Das D, Dey S, Brewster RC, Choubey S. Effect of transcription factor resource sharing on gene expression noise. PLoS Comput Biol. 2017;13(4):e1005491.

[42] Gillespie DT. Exact stochastic simulation of coupled chemical reactions. Journal of Physical Chemistry. 1977;81:2340-2361.

[43] Ghusinga KR, Dennehy JJ, Singh A. First-passage time approach to controlling noise in the timing of intracellular events. Proc Natl Acad Sci U S A. 2017;114(4):693-698. 
[44] Van Kampen N. Stochastic Processes in Physics and Chemistry. Third edition ed. Amsterdam: Elsevier; 2007.

[45] Redner S. A Guide to First-Passage Processes. Cambridge University Press; 2001.

[46] Iyer-Biswas S, Zilman A. First-Passage Processes in Cellular Biology. New York: Wiley; 2016.

[47] Savageau MA. Comparison of classical and autogenous systems of regulation in inducible operons. Nature. 1974;252:546-549. doi:10.1038/252546a0.

[48] Becskei A, Serrano L. Engineering stability in gene networks by autoregulation. Nature. 2000;405(6786):590-3.

[49] Phillips R, Kondev J, Theriot J, Garcia HG. Physical biology of the cell. 2nd ed. New York: Garland Science; 2013.

[50] Shreshtha M, Surendran A, Ghosh A. Estimation of mean first passage time for bursty gene expression. Phys Biol. 2016;13(3):036004.

[51] Burger A, Walczak AM, Wolynes PG. Influence of decoys on the noise and dynamics of gene expression. Phys Rev E Stat Nonlin Soft Matter Phys. 2012;86(4 Pt 1):041920.

[52] Hermsen R, Erickson DW, Hwa T. Speed, Sensitivity, and Bistability in Autoactivating Signaling Circuits. PLoS Comput Biol. 2011;7(11):e1002265.

[53] Elf J, Li GW, Xie XS. Probing transcription factor dynamics at the single-molecule level in a living cell. Science. 2007;316(5828):1191-4.

[54] Yu J, Xiao J, Ren X, Lao K, Xie XS. Probing gene expression in live cells, one protein molecule at a time. Science. 2006;311(5767):1600-3.

[55] Kennell D, Riezman H. Transcription and translation initiation frequencies of the Escherichia coli lac operon. J Mol Biol. 1977;114(1):1-21.

[56] Taniguchi Y, Choi PJ, Li GW, Chen H, Babu M, Hearn J, et al. Quantifying E. coli Proteome and Transcriptome with Single-Molecule Sensitivity in Single Cells. Science. 2010;329:533-538. 


\section{Figures}

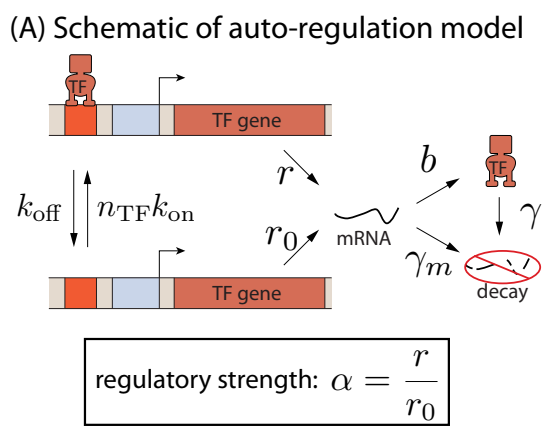

(C) Target gene in the model

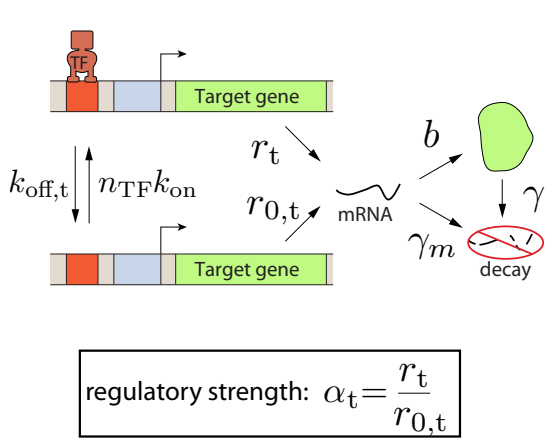

(B) Decoy binding sites in the model
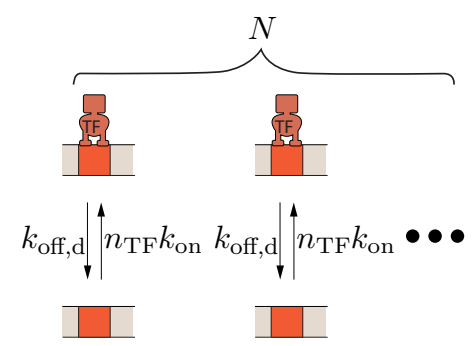

(D) First passage time for expression

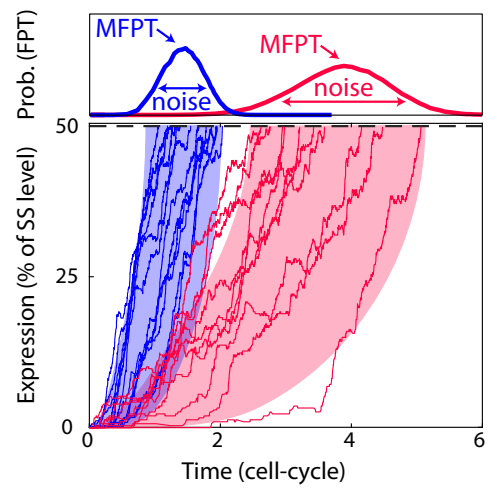

Figure 1: Mean first passage time in a self regulated gene. (A) Schematic of a self-regulating gene. (B) Schematic showing the reactions of TF binding/unbinding to competing decoy sites. (C) Reactions of a target gene regulated by the auto-regulatory TF gene. (D) First passage time is defined as the time for the gene expression to hit the threshold for the first time as shown here. For an auto-regulated gene, the mean first passage time (MFPT) and the distribution of FPT strongly depend on the strength of auto-regulation $(\alpha)$ and the TF-binding affinity. 

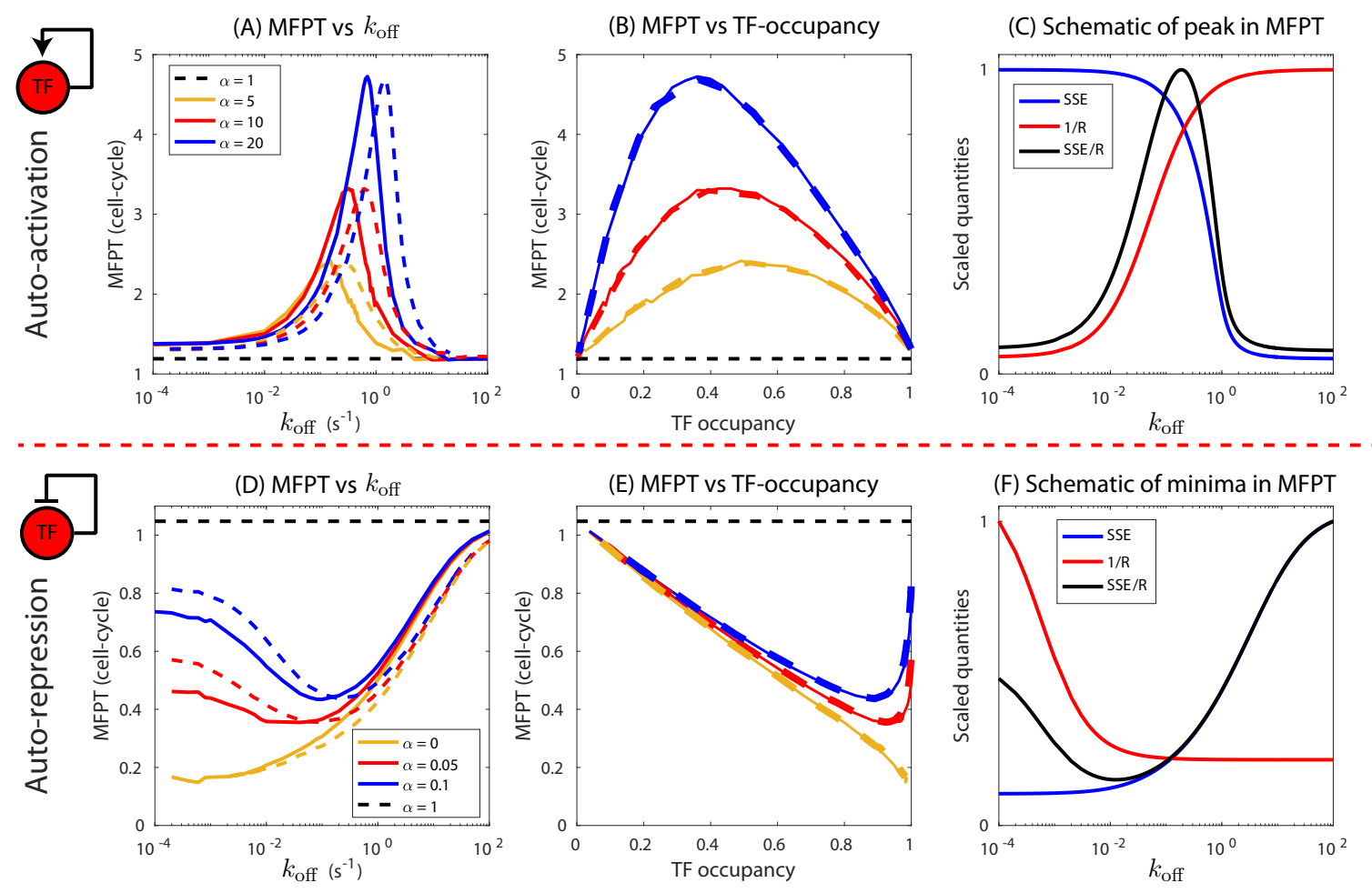

Figure 2: Effect of TF-binding affinity on MFPT for auto-regulated genes without any competing binding sites. (A, D) MFPT as a function of off-rate (binding affinity) of auto-activated gene and auto-repressed gene. Each curve (blue, red, yellow) is generated by keeping the transcription rate $\left(r_{0}\right)$ and translation rate $(b)$ fixed and varying only the binding affinity through $k_{\text {off }}$. Dashed curves correspond to doubling the translation rate $(2 b)$. Black dashed lines correspond to MFPT of a constitutive gene of transcription rate $r_{0}$. (B, E) MFPT as a function of TF-promoter occupancy. Solid and dashed curves are for translation rates $b$ and $2 b$, respectively. (C,F) Scaled steady state TF expression (SSE) and inverse of production rate $(1 / R)$ as a function of off-rate. The ratio of SSE and $R$ (black curve) qualitatively predicts the behavior of the MFPT as a function of off-rate. For auto-activation we use $r_{0}=0.0025 \mathrm{~s}^{-1}, b=0.025 \mathrm{~s}^{-1} \mathrm{mRNA}^{-1}$; for auto-repression $r_{0}=0.05, b=0.1 s^{-1} \mathrm{mRNA}^{-1}$. 

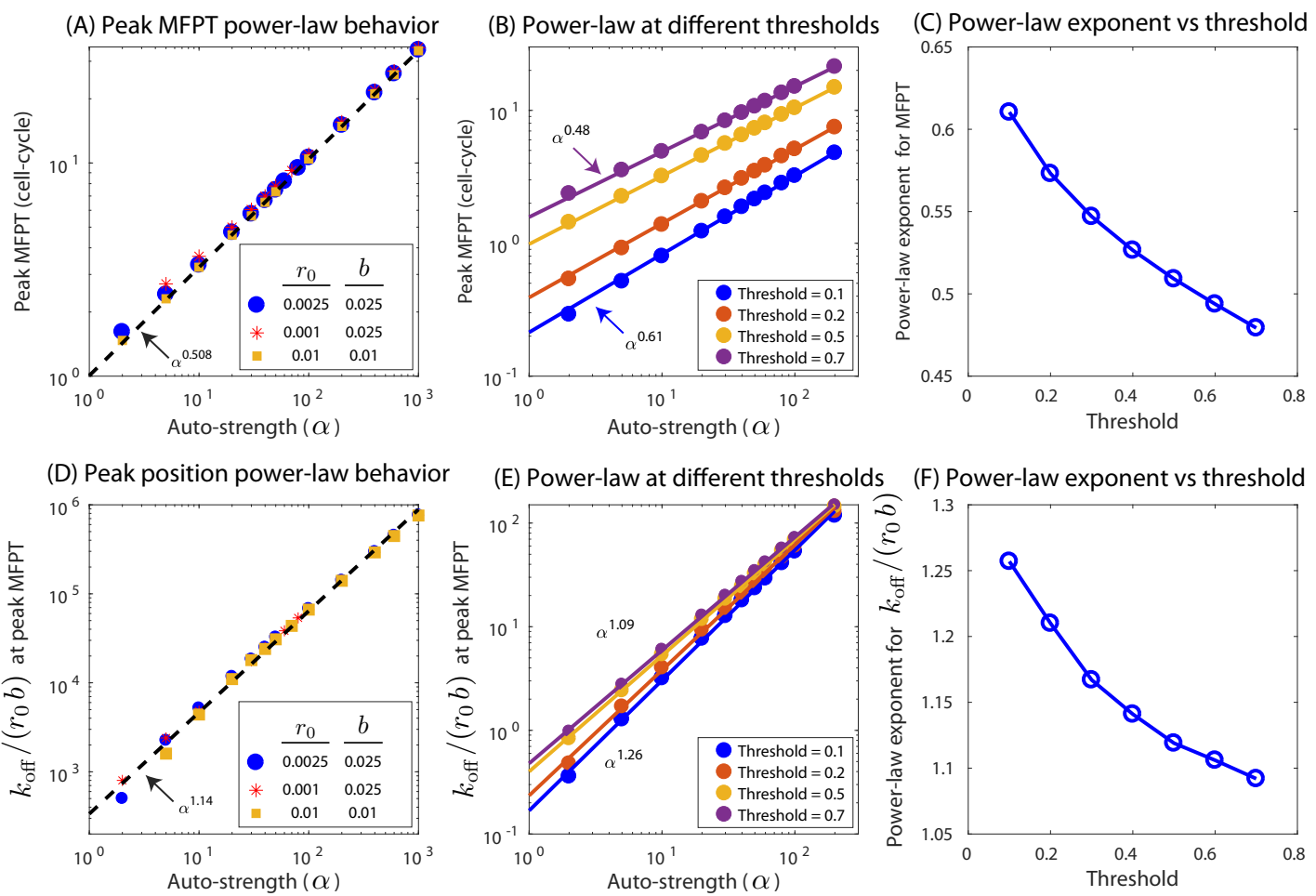

Figure 3: Power-law behavior in auto-activated gene. (A) Peak value of MFPT as a function of autoregulation strength, $\alpha$, shows a power-law behavior with approximate exponent of 0.5 irrespective of basal transcription or translation rates. (B) Peak MFPT for different expression thresholds have different exponents. (C)Power-law exponent of Peak MFPT versus auto-regulation strengths as a function of expression threshold. (D) The value of $k_{\text {off }} /\left(r_{0} b\right)$ at the peak MFPT also follows a power law behavior with exponent slightly larger than 1. (E) The exponent again depends on threshold. (F) The value of the exponent as a function of threshold. 
(A) MFPT vs decoy number
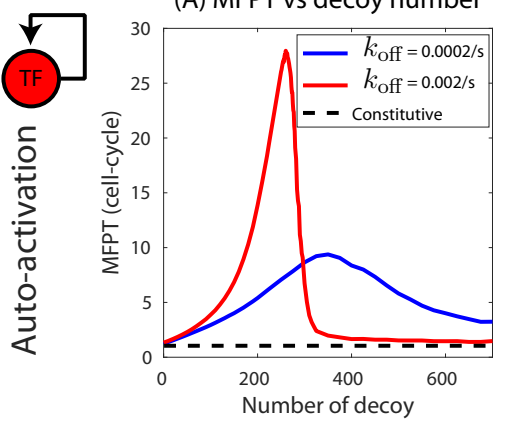

(D) MFPT vs decoy number

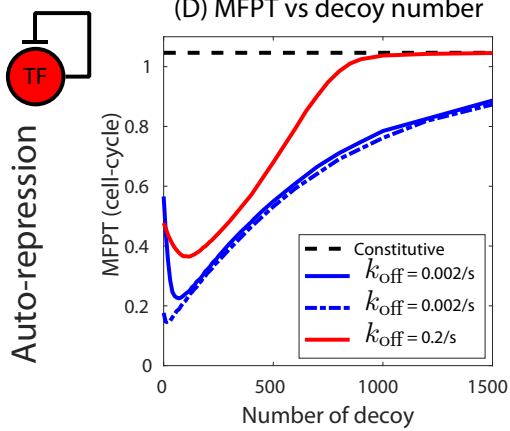

(B) MFPT vs TF-occupancy

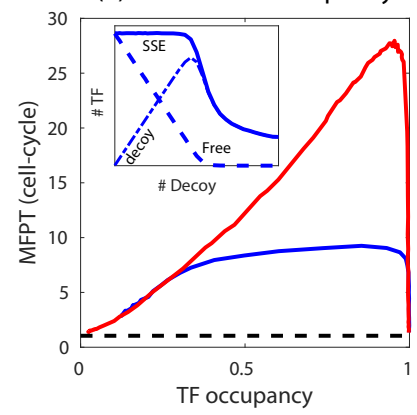

(E) MFPT vs TF-occupancy

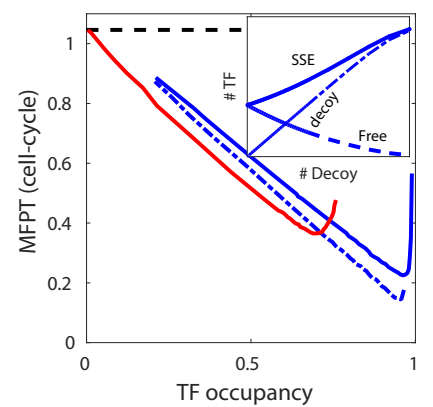

(C) MFPT space for $\alpha=10$

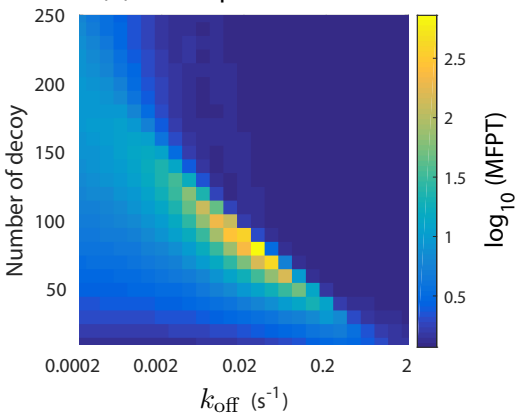

(F) MFPT space for $\alpha=0.1$

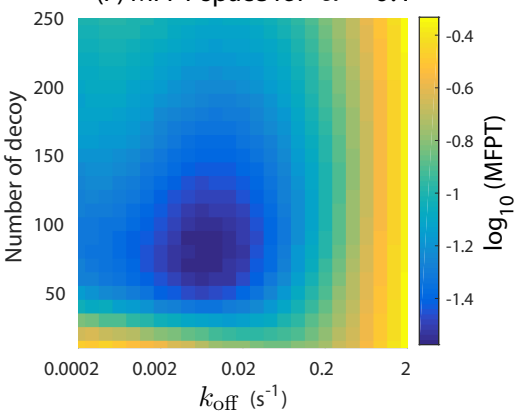

Figure 4: MFPT of an auto-regulated gene in a network of competing binding sites. The MFPT of an auto-activated gene as a function of (A) number of competitive binding sites and (B) steady-state occupancy of TF at the promoter when decoy number is varied. The red curve is for weaker TF binding while the blue curve is for stronger TF binding. Inset: MFPT as function of free TF in steady state. Black dashed lines correspond to MFPT of a constitutive gene of transcription rate $r_{0}$. (C) Heatmap showing phase space of MFPT in number of competing binding sites and affinity space for auto-activating gene $(\alpha=10)$. (D,E) The MFPT of an auto-repressed gene as a function of competing binding sites and steadystate occupancy. Dashed blue line correspond to complete repression $(\alpha=0)$. Inset: MFPT as function free TF in steady state. (F) Heatmap showing phase space of MFPT in number of competing binding sites and affinity space for auto-repressing gene $(\alpha=0.1)$. We use $\alpha=10, b=0.025 s^{-1} \mathrm{mRNA}^{-1}, r_{0}=0.0025 s^{-1}$ (activation) and $\alpha=0.1, b=0.05 s^{-1} \mathrm{mRNA}^{-1}, r_{0}=0.05 \mathrm{~s}^{-1}$ (repression). $\gamma=0.0003 / \mathrm{s}$ is used for protein degradation corresponding to $\tau=38.5 \mathrm{~min}$. 
(A) Auto-activation FPT distribution

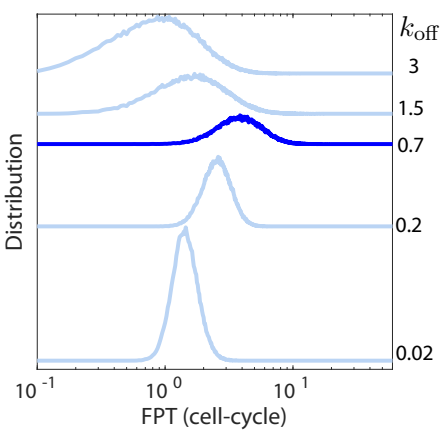

(D) Auto-activation FPT distribution

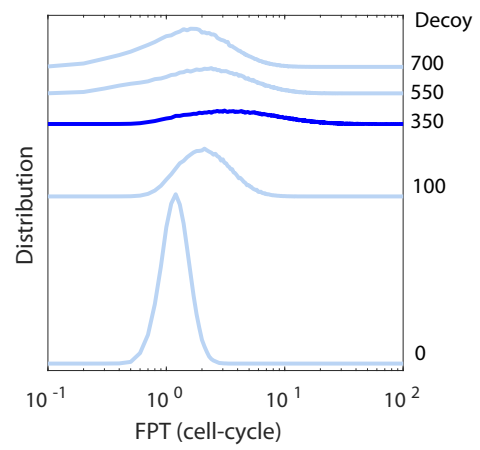

(B) Auto-repression FPT distribution

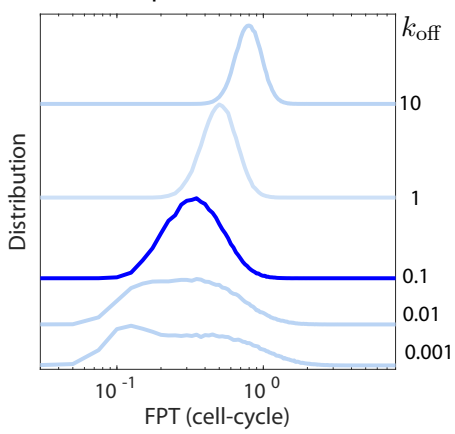

(E) Auto-repression FPT distribution

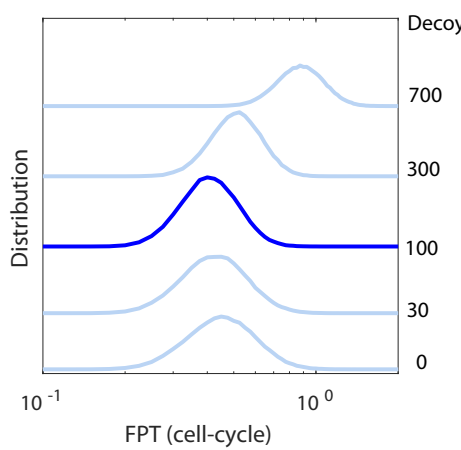

(C) Noise vs. MFPT as $k_{\text {off }}$ is tuned

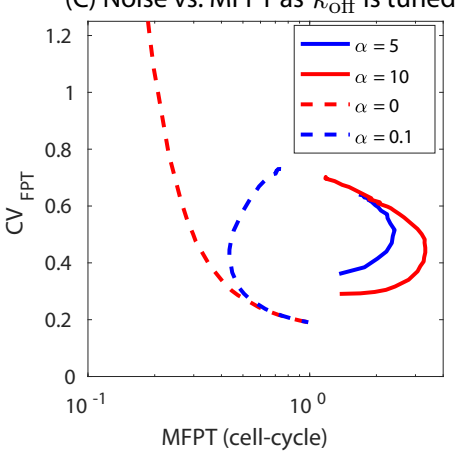

(F) Noise vs. MFPT as decoy \# is tuned

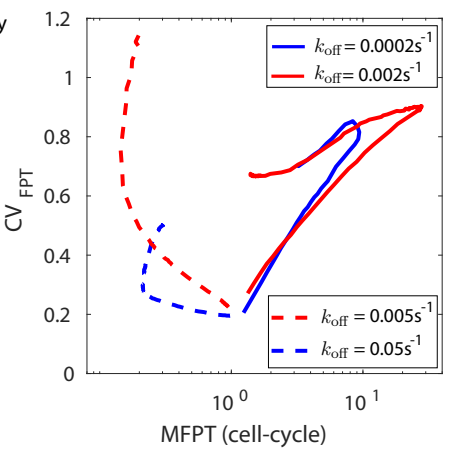

Figure 5: Distribution of first passage time (FPT) of an auto-activated TF gene. (A) The CV of first passage time as a function of MFPT when $k_{\text {off }}$ is changed to vary MFPT for auto-activation (solid lines) and auto-repression (dashed lines) of differing regulatory strength. (B-C) FPT distribution for several values of $k_{\text {off for }}(\mathrm{B})$ auto-activation and (C) auto-repression, the peak $k_{\text {off }}$ is shown in dark blue. Parts (D-F) show the same information but for varying decoy instead. 
(A) MFPT of activated target gene

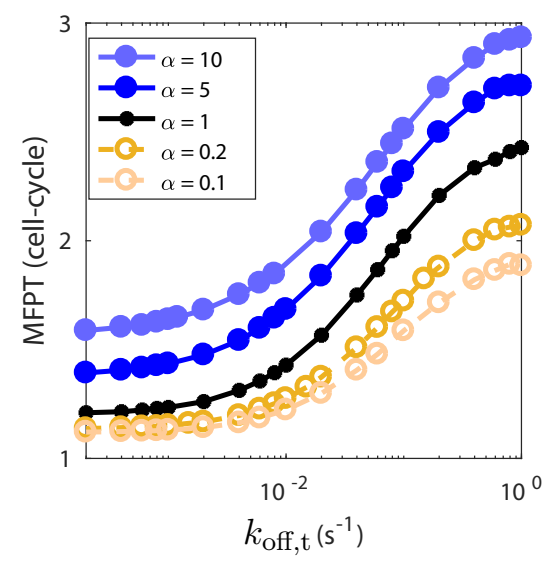

(C) MFPT of target gene vs TF gene

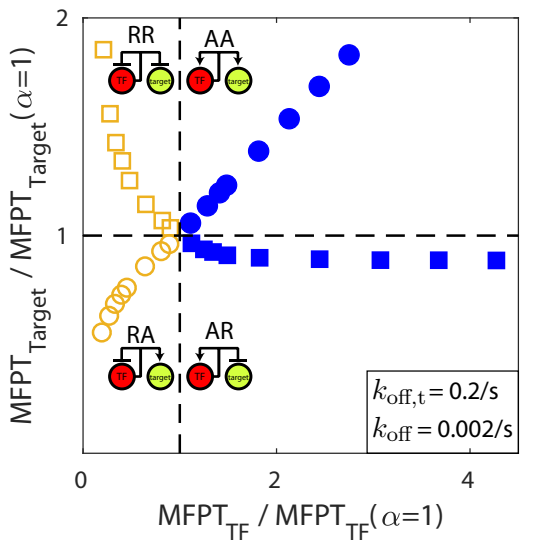

(B) MFPT of repressed target gene

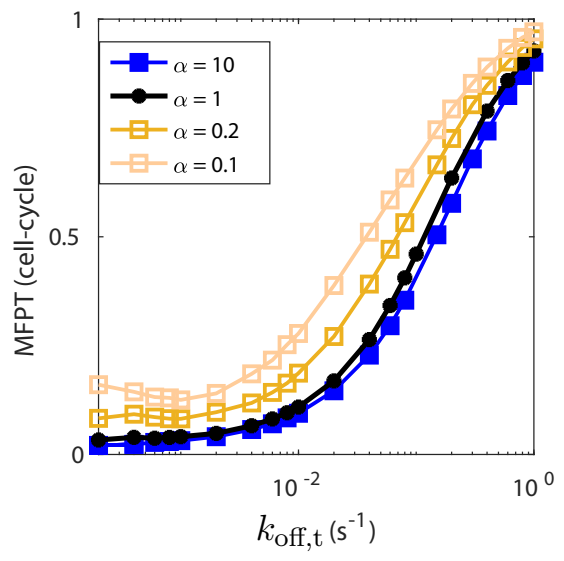

(D) Identical TF and target gene

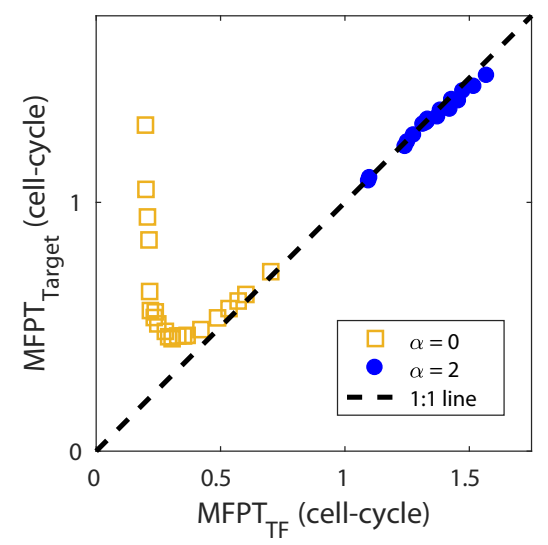

Figure 6: Expression timing of target gene is dictated by the nature of TF regulation. (A, B) MFPT of a target gene as a function of binding affinity of TF to the target gene $\left(k_{\mathrm{off}, \mathrm{t}}\right)$. Filled symbols represent the TF being auto-activated $(\alpha>1)$, open symbols represent when the TF gene is auto-repressed $(\alpha<1)$, and black asterisks are when the TF gene is constitutive $(\alpha=1)$. Circles represent target gene activation whereas squares represent target gene being repressed by the TF gene. (C) MFPT of target gene as a function of MFPT of TF gene. $\alpha$ is varied from from 0 to 50 while keeping $k_{\text {off }}=0.002 / s$ and $k_{\text {off }, \mathrm{t}}=0.2 / s$ constant. The MFPTs of TF and target genes are normalized by their respective MFPTs when $\alpha$ is one or equivalently when TF gene is constitutive. Translation rate of the TF gene is adjusted to achieve constant level of TF number $(\sim 50)$ for varying binding affinities and $\alpha$ in (A-C). (D) Plot showing asymmetry in the expression timing of TF and target gene when they have identical binding affinity, transcription and translation rates. Target gene repressed by an auto-repressed TF gene always has higher MFPT compared to the TF gene. Target gene activated by an auto-activated TF gene, on contrary, do not show any significant differences (target gene being moderately faster than the TF gene). 


\section{Supporting Figures}

(A) Effect of production rate on MFPT

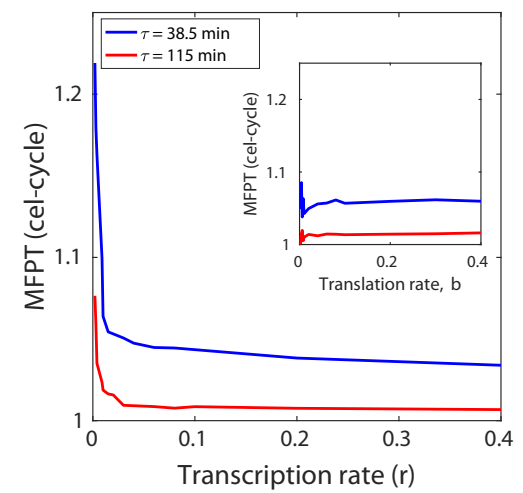

(B) Power law prediction for MFPT of constitutive gene

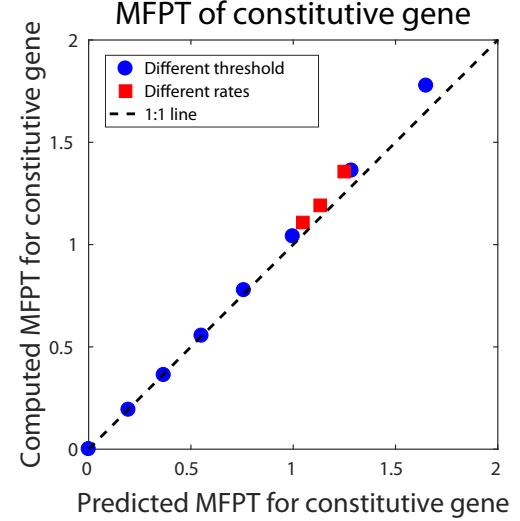

Figure S1: (A) Effect of transcription and translation rates on MFPT of a constitutive gene. At low transcription rates the MFPT of constitutive gene is usually higher than that at higher transcription rate. Inset: The translation rate on the other hand does not alter MFPT significantly. (B) Comparison of MFPT of a constitutive gene computed from simulation and predicted from the the power-law fit of peak MFPT versus $\alpha$ by setting $\alpha=1$. For the power-law fit data point corresponding to $\alpha=1$ is excluded, since $\alpha=1$ is a constitutive gene having constant MFPT as a function of $k_{\text {off }}$. 
(A) MFPT of auto-activation for different division rates

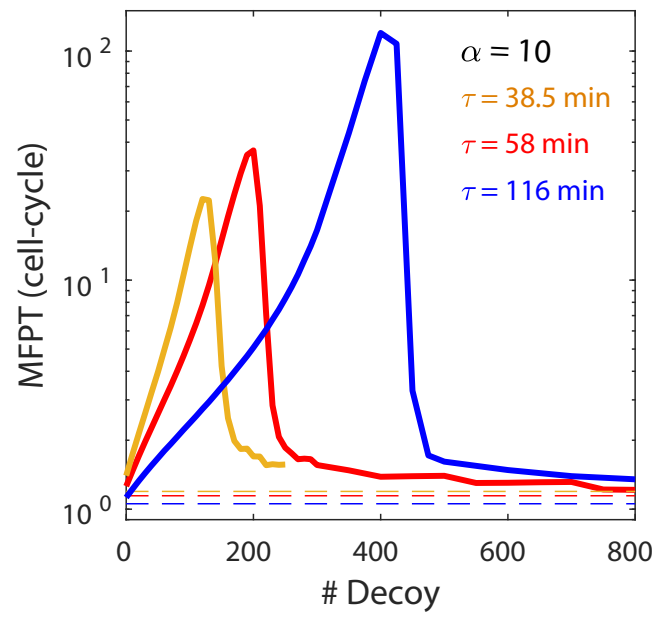

(C) MFPT of auto-activation for different division rates

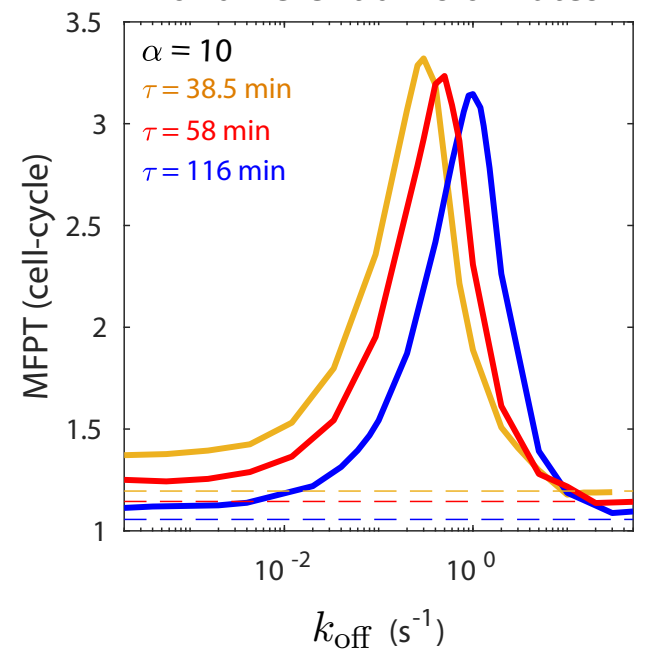

(B) MFPT of auto-repression for different division rates

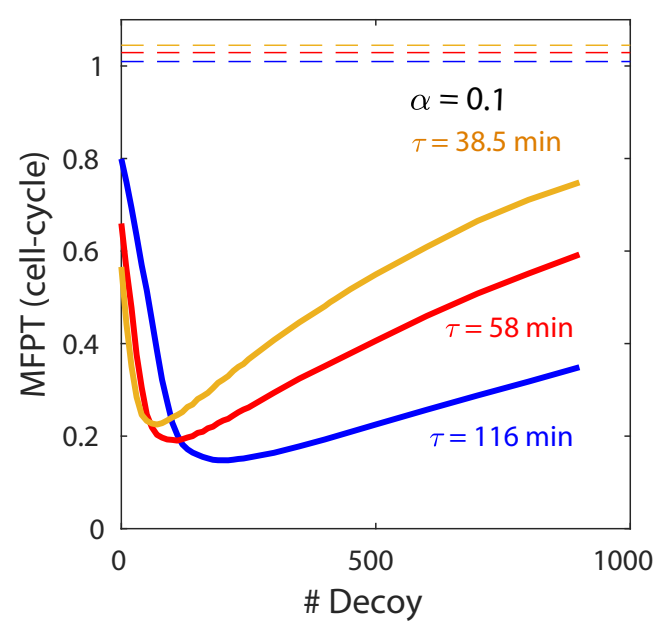

(D) MFPT of auto-repression for different division rates

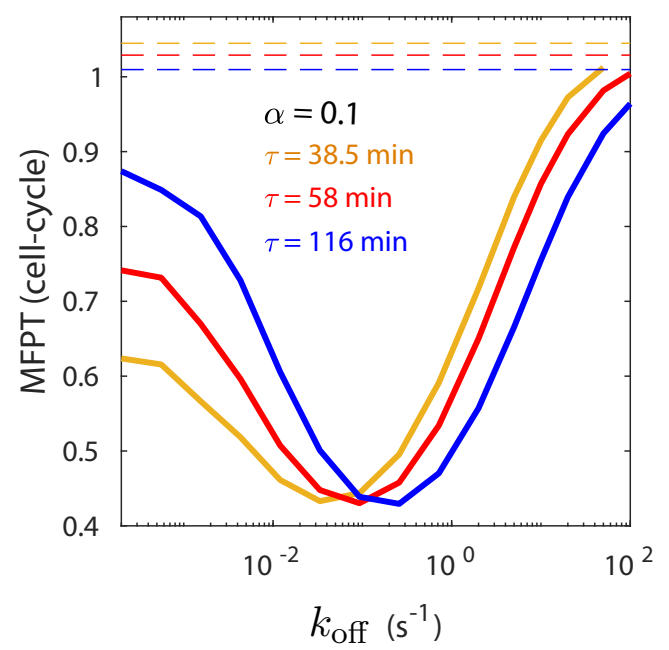

Figure S2: Effect of TF-binding affinity and competing binding sites on MFPT for auto-regulated genes for different cell division rates, $\tau=38.5 \mathrm{~min}$ (yellow), $58 \mathrm{~min}$ (red) and $116 \mathrm{~min}$ (blue). The TF gene is auto-activated with $(\mathrm{A}, \mathrm{C})$ or auto-repressed $(\mathrm{B}, \mathrm{D})$. Dashed lines correspond to MFPT of a constitutive gene of transcription rate $r_{0}$. Parameters used to generate the figure: $\alpha=10, r_{0}=0.0025 \mathrm{~s}^{-1}, b=$ $0.025 s^{-1} \mathrm{mRNA}^{-1}$ (auto-activation). $\quad \alpha=0.1, r_{0}=0.05 \mathrm{~s}^{-1}, b=0.05 \mathrm{~s}^{-1} \mathrm{mRNA}^{-1}$ (auto-repression). $k_{\text {off }}=0.002 s^{-1}$ is used for $(\mathrm{A}, \mathrm{B})$. 
(A) Peak MFPT power-law behavior

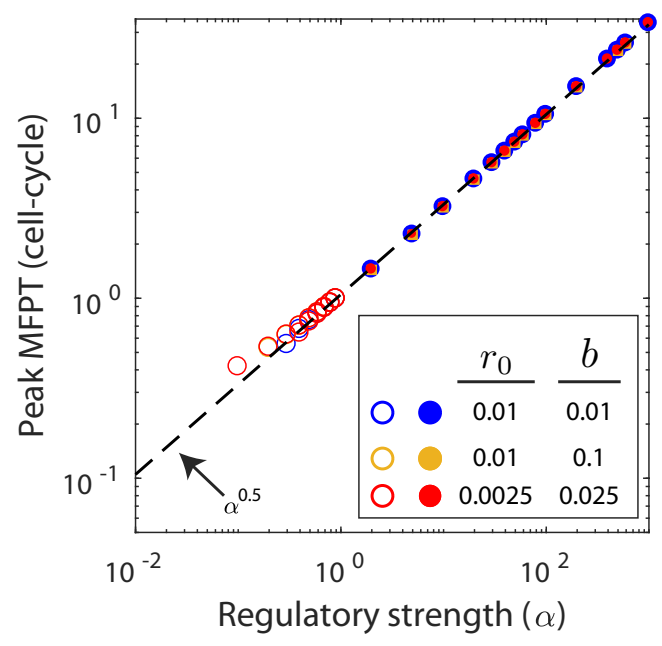

(C)

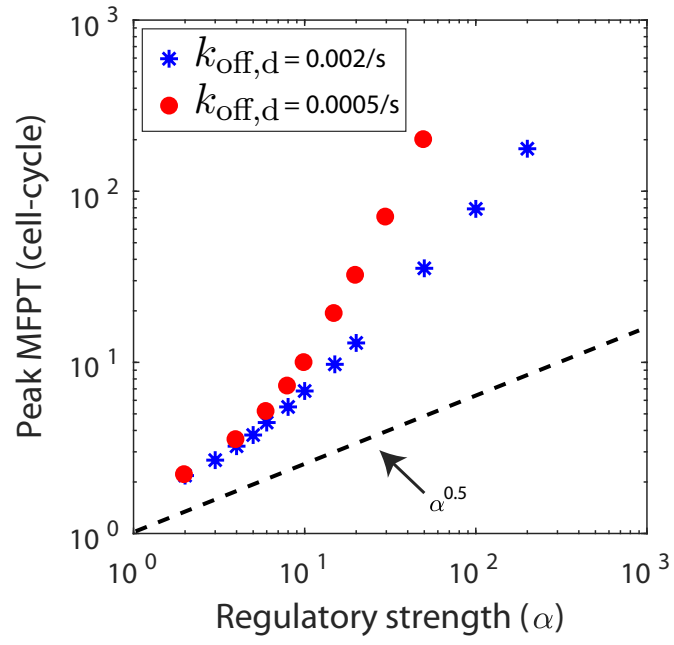

(B) Peak locaiton for different $\alpha$

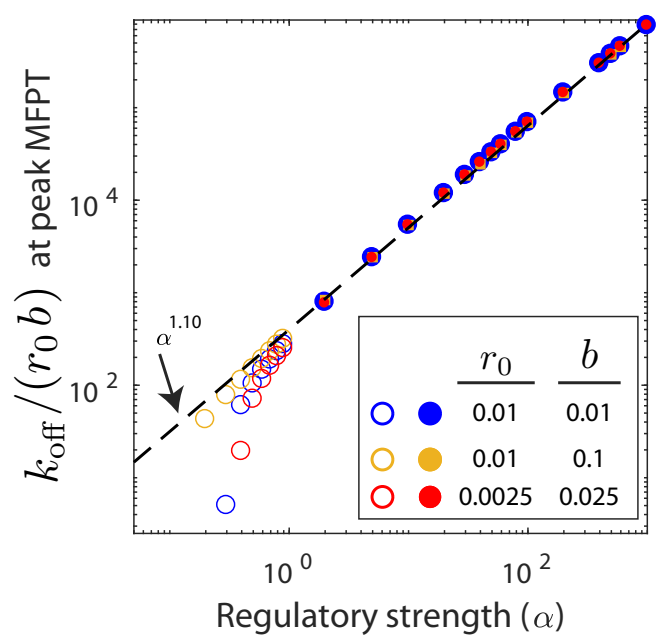

(D)

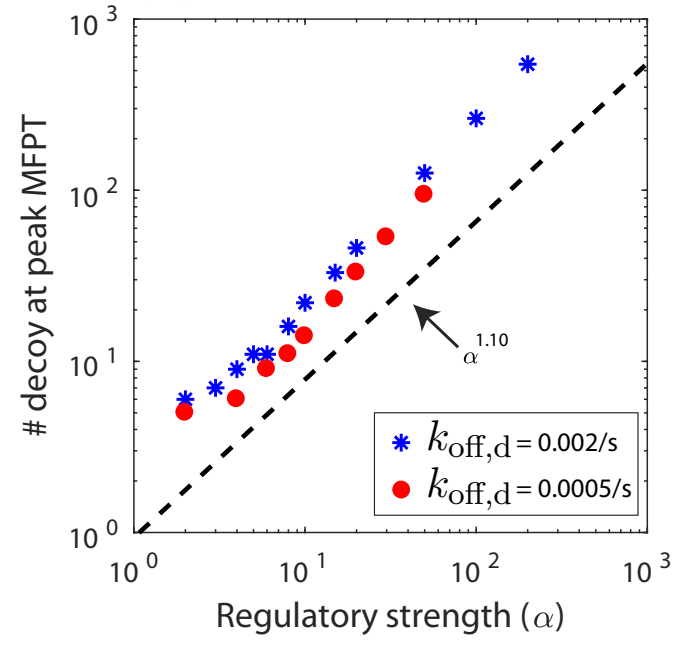

Figure S3: (A,B) Power-law behavior in self-repressing gene when affinity is varied. For an auto-repressing gene both the peak MFPT (A) and $k_{\text {off }}$ at the peak (B) deviate from the power-law fit as a function of auto-regulatory strength $\alpha$. Black dashed lines corresponds to power-law fit for auto-activating TF gene (i.e., $\alpha>1)$. Open circles are auto-activation $(\alpha>1)$ and filled circles are auto-repression $(\alpha<1)$. (C,D) Peak MFPT and number of decoys at the peak versus regulatory strength $(\alpha)$ when decoy is tuned. We do not observe any power-law behavior. Parameters: $k_{\mathrm{off}}=0.002 s^{-1}, r_{0}=0.001 s^{-1}, b=0.01 s^{-1} \mathrm{mRNA}^{-1}$. 
(A) MFPT of target gene vs TF gene

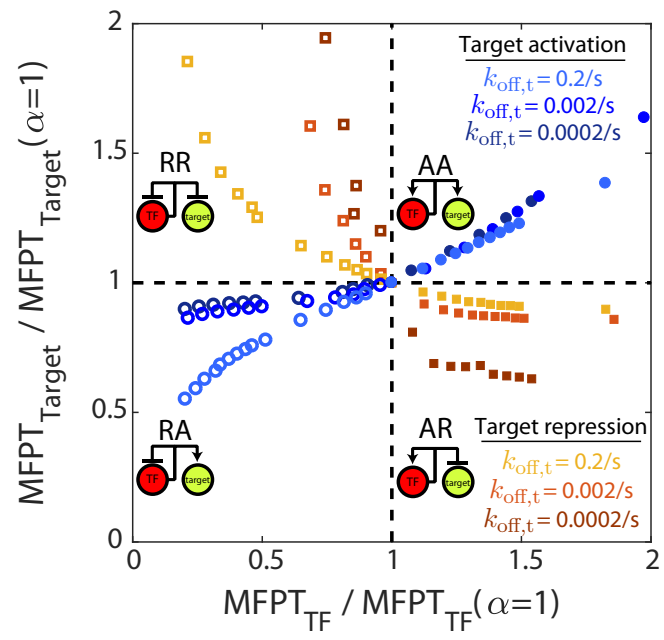

(B) Identical TF and target gene, vary decoy

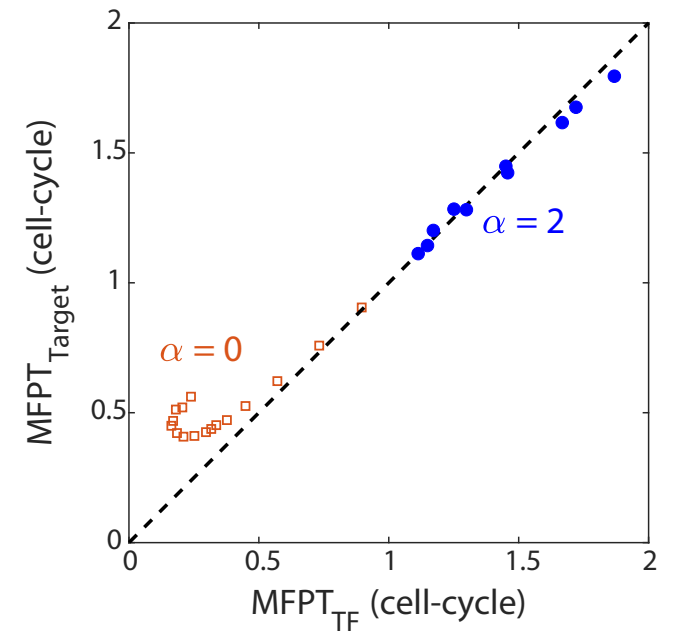

Figure S4: Expression timing of target gene depends on the nature of TF regulation. (A) MFPT of target gene as a function of MFPT of TF gene. $\alpha$ is varied from from 0 to 50 while keeping $k_{\text {off }}=0.002 s^{-1}$ and $k_{\mathrm{off}, \mathrm{t}}=0.0002 s^{-1}, 0.002 s^{-1}, 0.2 s^{-1}$ constant. The MFPTs of TF and target genes are normalized by their respective MFPTs when $\alpha$ is one or equivalently when TF gene is constitutive. Translation rate of the TF gene is adjusted to achieve constant level of TF number $(\sim 50)$. The TF gene is auto-activated (filled symbols) or auto-repressed (open symbols) and the target gene is activated (circles) or repressed (squares). (D) Plot showing asymmetry in the expression timing of TF and target gene when they have identical TFbinding affinity, transcription and translation rates. Decoy binding sites are varied along the curve from 0 to 300 for $\alpha=2$ (blue) and $\alpha=0$ (brown). 